\title{
Net energy gain in direct laser acceleration due to enhanced dephasing induced by an applied magnetic field
}

\author{
APL Robinson and AV Arefiev
}

\section{Published version information}

Citation: APL Robinson and AV Arefiev. "Net energy gain in direct laser acceleration due to enhanced dephasing induced by an applied magnetic field." Physics of Plasmas, vol. 27, no. 2 (2020): 023110.

DOI: $\underline{10.1063 / 1.5122893}$

This article may be downloaded for personal use only. Any other use requires prior permission of the author and AIP Publishing. This article appeared as cited above and may be found at DOI above.

This version is made available in accordance with publisher policies. Please cite only the published version using the reference above. This is the citation assigned by the publisher at the time of issuing the AAM. Please check the publisher's website for any updates. 


\section{Net energy gain in direct laser acceleration due to enhanced dephasing induced by an applied magnetic field}

A.P.L.Robinson ${ }^{1}$ and A. V. Arefiev ${ }^{2,3}$

1) Central Laser Facility, STFC Rutherford-Appleton Laboratory, Didcot, OX11 OQX,

United Kingdom

${ }^{2)}$ Department of Mechanical and Aerospace Engineering, University of California at San Diego, La Jolla, CA 92093, $U S A$

${ }^{3)}$ Center for Energy Research, University of California San Diego, La Jolla, CA, 92093,

USA

(Dated: 16 January 2020)

Even in the situation where an electron interacts with a single plane wave, the well-known dynamical adiabaticity can be broken when an applied magnetic field is present, which will act to increase the dephasing rate of the electron during the interaction. Here we demonstrate this for the case where there is a uniform static magnetic field which is oriented either parallel or perpendicular to the electric field of the incident plane wave, and perpendicular to the direction of its propagation. The described energy gain phenomenon has direct relevance to laser-plasma interactions that involve external magnetic fields generated by laser-driven capacitor coils.

\section{INTRODUCTION}

There is growing interest in the potential of mechanisms that might be broadly termed 'Direct Laser Acceleration' (DLA) ${ }^{1-16}$ for the generation of highly energetic electrons in ultra-intense laser-matter interactions ${ }^{17-19}$ In order to fully develop this class of mechanisms ${ }^{20-26}$ it is necessary to address those issues which any particle acceleration concept would have to address. In the case of DLA, there are a number of questions as to how net energy gain is achieved.

In certain particle acceleration schemes this is an obvious matter, but as DLA concerns electron acceleration in an electromagnetic wave (an oscillating system) this is actually not so straightfoward. Energy is periodically gained and lost with each cycle of oscillation, and if the adiabatic nature of this oscillation is not broken by some means then the electron may only gain an absolutely negligible amount of energy. Vacuum Laser Acceleration (VLA) faces essentially the same problem ${ }^{27-37}$. To be absolutely clear : net energy gain is the energy gained by the particle after the laser interaction has completely ceased, and this is quite distinct from the 'instantaneous' energy that the electron can acquire while the interaction with the laser pulse is on-going ${ }^{10}$. The other matter that we should probably clarify about this paper is that we are looking at DLA (as we and the cited papers understand the term) as a fast electron generation mechanism that occurs in near-critical plasmas. We do not consider it as a 'laser accelerator' scheme for the well-controlled acceleration of a defined bunch, comparable to LWFA or certain VLA schemes ${ }^{38}$

In previous work, the authors have looked at a few different possible routes to net energy gain. In Ref. [39], the authors showed that net energy gain can occur if the electron is already undergoing strong, relativistic oscillations in an external electric field when the laser pulse arrives, and in Ref. [40] the authors showed that the longitudinal component of a pulse of coherent dipole radiation can impart net energy to an electron.

One school of thought is that an interaction that involves more than a single plane wave is essential to achieving net energy gain via $\mathrm{DLA}^{9}$, or that multidimensional effects are required ${ }^{41}$. If this is the case, then the prospects for exploiting DLA in the case of a relatively weakly focussed laser pulse are rather limited. Part of the purpose of this paper is to show that this is not the case.

In this paper it is shown that when a static magnetic field acts to enhance the dephasing rate that this can directly cause net energy gain to occur, and that this can occur even with a perfect plane wave. Previously the authors showed that when a longitudinal field acts to reduce the dephasing rate between an electron and wavefronts, the electron is launched onto a superponderomotive trajectory ${ }^{10,42}$. It turns out, that increasing the dephasing rate can also have a profound effect, albeit in terms of energy absorption. To avoid confusion : we will show that these two results do not, in any way, contradict one another. The important distinction being between net energy gain (in this paper) and instantaneous energy during the interaction (previously in ${ }^{10}$ ).

The role of static magnetic fields is still poorly understood in the context of relativistic laser-plasma interactions, so this paper provides a valuable insight into how strong magnetic fields impact electron acceleration. Previously, this topic raised relatively little interest because of technological limitations to achieve relevant field strengths. However, significant progress has been made over the recent years in generating strong magnetic fields with laser-driven coils using ns-long laser pulses ${ }^{43,44}$. Often these are referred to as 'capacitor-coil' targets ${ }^{45}$. These fields reach hundreds of Tesla in strength and persist on a ns time-scale while occupying a volume that is hundreds of microns in dimensions. In the context of the considered problem, such fields can indeed be considered 
as static and uniform, thus justifying our approximation. It has already been shown that the fields of this magnitude can meaningfully impact relativistic laser-plasma interactions ${ }^{44,46}$, but their role on electron acceleration has not been examined, which strongly motivated this work. We note that, at a number of facilities, it would be possible to exploit the presence of both long and short pulse beamlines to simulataneously generate such strong quasi-static B-fields and examine their effect on the shortpulse interaction. The parameters used in the calculations presented are for optical wavelengths, and the corresponding laser intensity is easily achievable on current laser facilities. We also note that DLA appears to have been observed in experiments using quite modest laser parameters $^{47}$.

The paper is organized as follows: In Sec. II we show how enhanced dephasing can, in principle, lead to net energy gain. In Sec. III we consider a specific situation where this principle can apply $\left(B_{\text {ext }} \| E_{y}\right)$, and in Sec. IV we provide a numerical demonstration of net energy gain in this scenario. In Sec. V we consider another specific situation $\left(B_{e x t} \perp E_{y}\right)$ that is somewhat different but which also demonstrates net energy gain, and in Sec. VI we provide a numerical demonstration of this.

\section{GENERAL THEORY}

Consider the situation in which the $E_{y}$ component of an incident plane electromagnetic wave is either the sole or leading term in the energy equation of a single electron moving in this plane wave, i.e.

$$
\frac{d \gamma}{d t}=-\frac{e E_{y} v_{y}}{m_{e} c^{2}}
$$

The techniques that have previously been applied to this class of problems can be applied here. This starts by defining $\tau=t-x / c$, writing the laser vector potential as $A_{y}=A(\tau)=A_{0} \cos \omega_{L} \tau$, and thus writing $E_{y}=-d A / d \tau$. If we also define,

$$
R=\gamma \frac{d \tau}{d t}
$$

and we assume, $p_{y}=e A$, then we can transform Eq. 1 into,

$$
\frac{d \gamma}{d t}=\frac{a}{R} \frac{d a}{d t}
$$

where $a=e A / m_{e} c$. This can now be integrated by parts to obtain

$$
\gamma=\gamma_{0}+\frac{a^{2}}{2 R}+\int \frac{a^{2}}{2 R^{2}} \frac{d R}{d t} d t
$$

This expression is quite revealing, when one considers what happens after the laser pulse has overtaken the electron and the interaction has ceased. The first term on the right hand side is the initial $\gamma$ of the electron, which will be $\gamma_{0}=1$ for electrons initially at rest. The second term on the right hand side will vanish, as $a \rightarrow 0$ as the laser pulse overtakes the electron. Therefore, without the third term, there is no prospect of net energy gain. The third term is not necessarily zero, and thus there is the prospect of net energy gain provided that $d R / d t \neq 0$ at least for some time during the interaction. Note that the first two terms of Eq. 4 completely reproduce a result obtained previously by the authors ${ }^{10}$, namely that the energy during the laser interaction can be enhanced by reduced dephasing.

When we consider the third term in more detail we find that it is rather interesting. Since $a^{2} / R^{2}$ is positive, net energy gain only when there is a dominant interval where $d R / d t>0$, i.e. only when the dephasing rate is increasing. Therefore, the act of increasing the dephasing rate, actually has profound consequences not appreciated in our previous studies.

We have arrived at this result via a somewhat mathematical route, however there is a qualitative way to look at this as well. The work done on the electron is $\propto E_{\perp} v_{\perp}$. If we consider only the laser field, then both $E_{\perp}$ and $v_{\perp}$ are periodic functions that are $\pi / 2$ out of phase with one another. This means that energy gained in one half-cycle will be lost in the next half-cycle, and when the laser pulse overtakes the electron it will then return to rest. The only way to break this is for the electron to experience acceleration or deceleration by another field (not the driving laser pulse) during the interaction as this will shift the $E_{\perp}$ experienced by the electron relative its $v$ and thus break the cancellation between subsequent halfcycles. Looked at this way, the natural way would be to supply a transverse B-field so there would be an additional ' $\mathrm{v} \times \mathrm{B}$ ' force in the direction of laser propagation (but without any work being done on the electron).

\section{THE $B_{e x t} \| E_{y}$ SCENARIO}

Let us now look at the possibility of net energy gain in the situation where a plane wave interacts with a single electron in the presence of a magnetic field that is parallel to the electric field of the laser pulse. The case where the magnetic field is parallel to the direction of EM wave propagation has been extensively analyzed in the literature $\left(\mathrm{see}^{48}\right)$. However the problem considered here has received much less attention. This problem was considered by Angus and Krashennikov ${ }^{49}$, however they concluded that there should be negligible net energy gain in this configuration. This result is only true when the pulse duration is longer than the cyclotron period, and when this restriction is removed, net energy gain is quite possible, as we shall show.

For the purposes of this analysis the electric field com- 
ponent of the laser is in the $\mathrm{y}$-direction, and the applied magnetostatic field is $\mathbf{B}_{s}=B_{0} \hat{\mathbf{y}}$. The governing equations are thus,

$$
\begin{aligned}
\frac{d \gamma}{d t} & =\frac{e v_{y}}{m_{e} c^{2}} \frac{d A}{d \tau} \\
\frac{d p_{x}}{d t} & =\frac{e v_{y}}{c} \frac{d A}{d \tau}+e v_{z} B_{0} \\
\frac{d p_{y}}{d t} & =e\left(1-\frac{v_{x}}{c}\right) \frac{d A}{d \tau} \\
\frac{d p_{z}}{d t} & =-e v_{x} B_{0}
\end{aligned}
$$

The $p_{y}$ equation can be immediately integrated to yield, $p_{y}=e A$. This problem therefore meets the condition that the energy equation only includes the contribution due to the laser's electric field, and that $p_{y}=e A$ and this is therefore an ideal case for looking at net energy gain due to increasing dephasing. Using this the remaining equations can be re-cast as follows,

$$
\begin{aligned}
R \frac{d R}{d \tau} & =-\Omega \tilde{p}_{z} \\
R \frac{d \tilde{p}_{x}}{d \tau} & =a \frac{d a}{d \tau}+\Omega \tilde{p}_{z} \\
R \frac{d \tilde{p}_{z}}{d \tau} & =-\Omega \tilde{p}_{x}
\end{aligned}
$$

where the tilde denotes a momentum normalized by $m_{e} c$,

$$
\Omega=e B_{0} / m_{e},
$$

is the classical gyrofrequency and $a=e A / m_{e} c$ is a normalized vector potential. From this set of equations we can therefore arrive at a few conclusions without detailed calculation. Firstly let's assume that the laser duration is shorter than the gyroperiod. In this case, to a reasonable approximation, the effect of the laser will be to produce positive $p_{x}$ (since $p_{x}=m_{e} c a^{2} / 2$ in the case without the magentic field), which implies that, at least initially we will produce negative $p_{z}$. In turn this will cause $R$ to continuously increase during this period. Provided the lase interaction only occurs over this period, we should get net energy gain which is unambiguously due to enhanced dephasing.

In order to ensure that the laser interaction only occurs in this period, we need to ensure that the laser duration is less than half a gyroperiod. This implies that we require that,

$$
\tau_{L}<\frac{\pi \gamma_{*} m_{e}}{e B_{0}}
$$

where $\gamma_{*}$ is the characteristic relativistic factor during the interaction. Due to the decreasing dephasing, it can be lower that $\gamma=1+a_{0}^{2} / 2$ that the electron reaches without the magnetic field, where $a_{0}=\max (a)$.

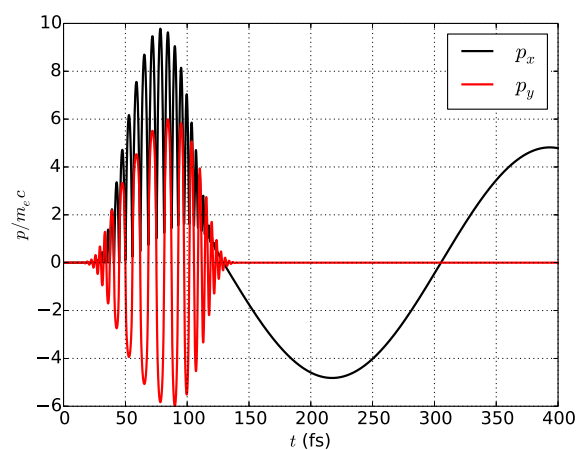

FIG. 1. Plot of $p_{x}(t)$ and $p_{y}(t)$ in numerical demonstration of $B_{\text {ext }} \| E_{y}$ problem.

\section{NUMERICAL DEMONSTRATION OF THE $B_{\text {ext }} \| E_{y}$ SCENARIO}

Numerical calculations of the problem discussed in Sec. III were carried out. The numerical calculations are done by integrating the primitive forms of the equations of motion for a single electron with a specified laser field. The integration algorithm used for this is the second order method of Boris. The normalized vector potential had the form,

$$
a_{y}=a_{0} \cos \omega_{L} \tau \exp \left[-\frac{\left(\tau-\tau_{0}\right)^{2}}{2 \tau_{L}^{2}}\right],
$$

where we set $\tau_{0}=5 \tau_{L}$ for the calculations that follow. For the baseline calculation we set $a_{0}=6, \tau_{L}=10 \mathrm{fs}$, $\lambda_{L}=1 \mu \mathrm{m}$ (laser wavelength). The electron is initially at rest at $x=y=z=0$. The time step was set to $0.025 \mathrm{fs}$, which is substantially less than the time step required even accounting for the magnitude of the vector potential ${ }^{50}$. The applied magnetostatic field was set to $B_{0}=500 \mathrm{~T}$. If we make approximate $\gamma_{*} \approx a_{0}$, then for this value of $B_{0}$, we have $\pi \gamma_{*} m_{e} / e B_{0}=215 \mathrm{fs}$ (see Eq. 13 ), so choosing $\tau_{L}=10$ fs should ensure that the entire interaction is limited to the first half gyroperiod as we require.

The results of this calculation are shown in fig.s 1-3. In fig. 1 we show how $p_{x}$ and $p_{y}$ evolve in this calculation. As expected, $p_{y}$ is set by the vector potential of the laser pulse, and returns to zero once the laser pulse overtakes the electron. The evolution of $p_{x}$, on the other hand, is a very different matter. Initially this roughly follows the pure plane-wave solution $\left(\tilde{p}_{x}=a^{2} / 2\right), p_{x}$ also acquires a much more slowly-varying component that manifests after the pulse overtakes the electron. During the interaction, $p_{x}$ is positive. We therefore immediately see from fig. 1 that net energy gain occurs in this calculation.

In fig. 2 the evolution of $p_{z}$ is plotted alongside that of 


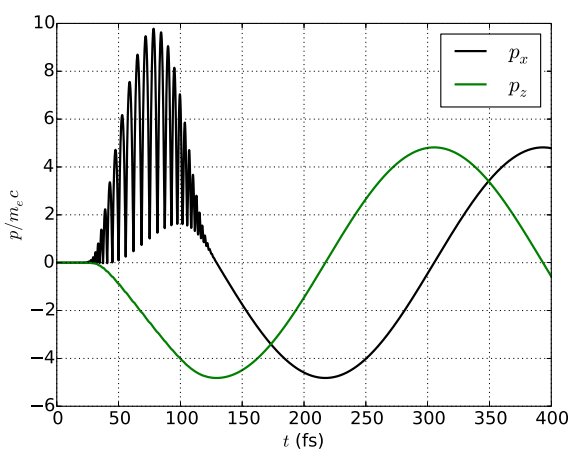

FIG. 2. Plot of $p_{x}(t)$ and $p_{z}(t)$ in numerical demonstration of $B_{e x t} \| E_{y}$ problem.

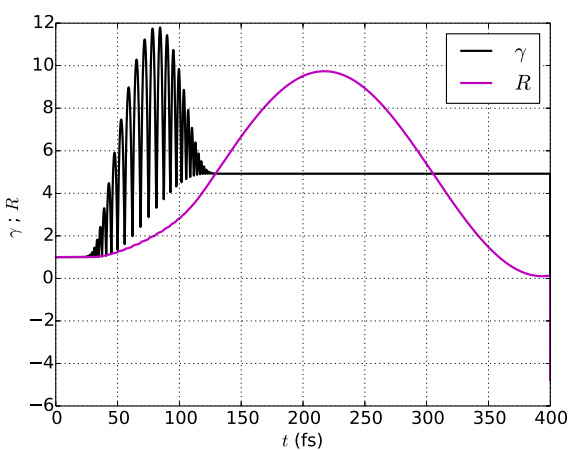

FIG. 3. Plot of $\gamma(t)$ and $R(t)$ in numerical demonstration of $B_{\text {ext }} \| E_{y}$ problem.

$p_{x}$. Here we see that as $p_{x}$ acquires a positive value (during the interaction), $p_{z}$ acquires a negative value. This is precisely what we had earlier inferred by examining the equation set.

Finally in fig. 3 the evolution of $\gamma$ and $R$ are plotted. Here we see, as expected, that the evolution of $\gamma$ shows net energy gain. However it is also seen that this occurs as $R$ is continuously increasing, thus demonstrating that net energy gain occurs when $R$ increases.

As expected, there is an optimal magnetic field for achieving the maximal energy retention by the electron. Figure 4 shows $\gamma_{e n d}$, which is the $\gamma$-factor after the interaction with the laser pulse is over, as a function of $\Omega$ for two different laser pulse durations. If $\gamma_{*} / \Omega \gg \tau_{L}$, then the magnetic field is too weak to manifest itself over the time associated with the electron-laser interaction. On the other hand, the reduction in the electron dephasing

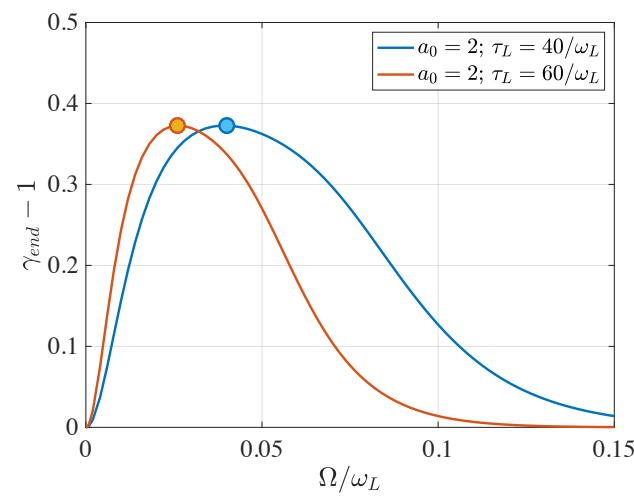

FIG. 4. Plot of final $\gamma$, denoted as $\gamma_{e n d}$, as a function of the magnetic field strength for the scenario with $B_{e x t} \| E_{y}$. The optimal field corresponds to $\Omega=0.04 \omega_{L}$ for $\tau_{L}=40 / \omega_{L}$ and to $\Omega=0.026 \omega_{L}$ for $\tau_{L}=60 / \omega_{L}$.

that occurs for $\gamma_{*} / \Omega<\tau_{L}$ causes $\gamma_{\text {end }}$ to drop. The optimal magnetic field strength decreases for the longer pulse, which is consistent with our explanation of the underlying mechanism.

The electron trajectory for the optimal magnetic field at $\tau_{L}=40 / \omega_{L}$ shown in Fig. 5 (upper panel) confirms that the electron performs only half of the rotation in the $(x, z)$-plane. The electron trajectory after the interaction is a circle, representing residual gyromotion caused by the interaction with the laser pulse. The lower panel shows the evolution of the electron momentum over the same trajectory. During the laser pulse, the electron moves along trajectory segments that are similar to parabolas in the $\left(p_{x}, p_{y}\right)$-plane over one laser oscillation.

\section{THE $B_{e x t} \perp E_{y}$ SCENARIO}

Now we can consider a closely related problem, where the applied magnetostatic field is perpendicular to both the electric field of the laser and the wavevector of the laser, i.e. $\mathbf{B}_{e x t}=B_{0} \hat{\mathbf{z}}$. Using the same procedure employed in Sec. III we can cast the governing equations in the following form

$$
\begin{aligned}
R \frac{d R}{d \tau} & =\Omega \tilde{p}_{y}, \\
R \frac{d \tilde{p}_{x}}{d \tau} & =\tilde{p}_{y} \frac{d a}{d \tau}-\Omega \tilde{p}_{y}, \\
R \frac{d \tilde{p}_{y}}{d \tau} & =R \frac{d a}{d \tau}+\Omega \tilde{p}_{x} .
\end{aligned}
$$

We immediately see that $p_{y} \neq e A$. What is therefore of additional interest in this scenario is that the condition $p_{y}=e A$ is relaxed in this case. However the energy equation still only involves the $E_{y}$ term. Importantly we 

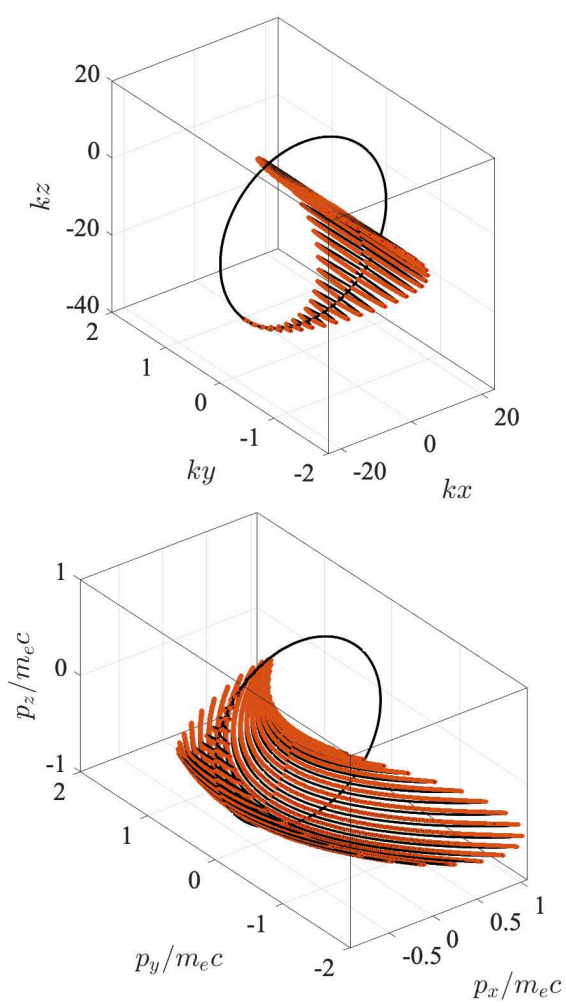

FIG. 5. Electron trajectory (upper) and electron momentum (lower) for $\tau_{L}=40 / \omega_{L}$ and $\Omega=0.04 \omega_{L}$ for the scenario with $B_{\text {ext }} \| E_{y}$. The set of parameters corresponds to the blue circle in Fig. 4. The red color indicates the electron energy gain from the laser pulse. The wave vector $k$ is defined as $k=2 \pi / \lambda_{L}$

see that the dephasing rate will evolve due to $p_{y}$ which must evolve as it is being driven by the laser pulse. Without detailed calculation, it would appear that net energy gain is therefore possible. However it will rely on $p_{y}$ acquiring a slow positive bias during the interaction. For a short laser pulse (see Eq. 13) we would expect $p_{y}$ to rise at the start of the interaction as a gyro-orbit is driven impulsively by the laser (by analogy with an impulsively driven harmonic oscillator). This will cause $R$ to increase in the first half-cycle of, and we can expect net energy gain provided (again) that the interaction is confined to the first half-cycle of the gyro-orbit. In the following section we will see that this is confirmed by full numerical calculations.

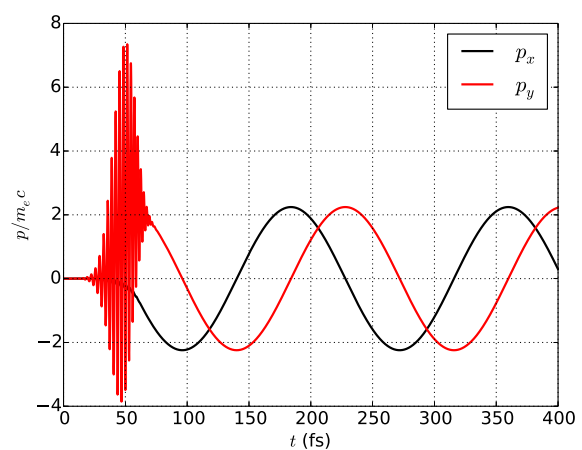

FIG. 6. Plot of $p_{x}(t)$ and $p_{y}(t)$ in numerical demonstration of $B_{\text {ext }} \perp E_{y}$ problem.

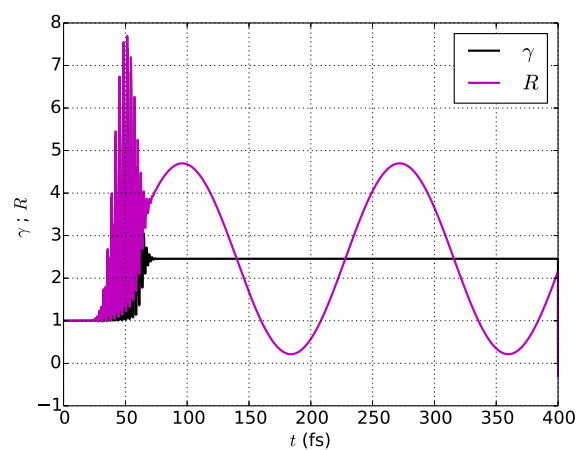

FIG. 7. Plot of $\gamma(t)$ and $R(t)$ in numerical demonstration of $B_{\text {ext }} \perp E_{y}$ problem.

\section{NUMERICAL DEMONSTRATION OF THE $B_{\text {ext }} \perp E_{y}$ SCENARIO}

Numerical calculations of the problem discussed in Sec. $\mathrm{V}$ were carried out using a method almost identical to that used in Sec. IV (except for the change to the applied magnetostatic field). The results of this are shown in fig.s 6 and 7.

In fig. 6 the evolution of $p_{x}$ and $p_{y}$ in this calculation are plotted. The interaction with the laser pulse ceases after about $75 \mathrm{fs}$, after which we see from the continued gyrations in the magnetostatic field that significant net energy gain has occurred. This is confirmed in fig. 7 in which $\gamma$ and $R$ are plotted, and this also shows that this coincides, as predicted, by a continuous increase in $R$.

In this case there is also an optimal magnetic field for achieving the maximal energy retention by the electron. 


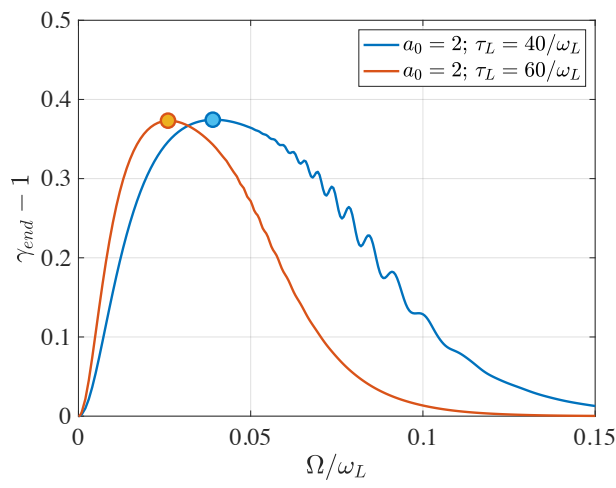

FIG. 8. Plot of final $\gamma$, denoted as $\gamma_{e n d}$, as a function of the magnetic field strength for the scenario with $B_{e x t} \perp E_{y}$. The optimal field corresponds to $\Omega=0.039 \omega_{L}$ for $\tau_{L}=40 / \omega_{L}$ and to $\Omega=0.026 \omega_{L}$ for $\tau_{L}=60 / \omega_{L}$.

The corresponding Fig. 8 closely resembles Fig. 4 where $\gamma_{e n d}$ is shown for the case with $B_{e x t} \| E_{y}$. In fact, the optimal field strength for $B_{\text {ext }} \perp E_{y}$ is almost identical to that for $B_{e x t} \| E_{y}$, provided that the laser pulse duration and its peak amplitude are the same. The electron trajectory and the electron momentum for the optimal magnetic field at $\tau_{L}=40 / \omega_{L}$ are shown in Fig. 9. The only difference with the previous cases is that there is no motion along the $z$-axis. The key features of mechanism however remain unaffected.

\section{LONGITUDINAL ELECTRIC FIELD}

The most obvious way to enhance dephasing is for the electron to encounter decelerating electric field aligned with the wavevector of the laser pulse. However since this electric field also does work on the electron, which counteracts forward acceleration, the ability for this to produce net energy gain is very limited. This can be shown as follows : The net energy change in this case can be written as,

$$
\gamma=\gamma_{0}+\frac{a^{2}}{2 R}+\int \frac{a^{2}}{2 R^{2}} \frac{d R}{d t} d t-\int \frac{e E_{x} v_{x}}{m_{e} c^{2}} d t
$$

Recall that,

$$
\frac{d R}{d t}=\frac{e E_{x}}{m_{e} c} \frac{R}{\gamma},
$$

and by substituting this we arrive at,

$$
\gamma=\gamma_{0}+\frac{a^{2}}{2 R}+\int \frac{e E_{x}}{m_{e} c}\left(\frac{a^{2}}{2 R \gamma}-\frac{v_{x}}{c}\right) d t .
$$
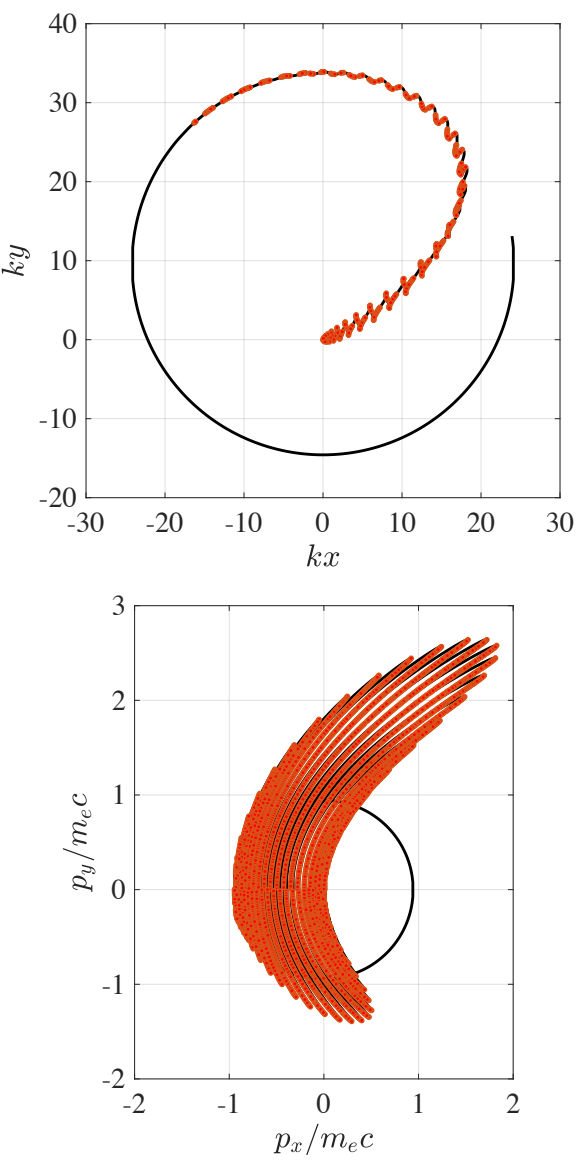

FIG. 9. Electron trajectory (upper) and electron momentum (lower) for $\tau_{L}=40 / \omega_{L}$ and $\Omega=0.039 \omega_{L}$ for the scenario with $B_{e x t} \perp E_{y}$. The set of parameters corresponds to the blue circle in Fig. 8. The red color indicates the electron energy gain from the laser pulse. The wave vector $k$ is defined as $k=2 \pi / \lambda_{L}$.

In the limit where $v_{x} \rightarrow c$, since $\gamma \approx a^{2} / 2 R$, the two terms in the integrand will cancel, and thus there will be no net energy gain. In the non-relativistic limit $\left(v_{x} \ll c\right)$, the integrand does not cancel, but is $\propto a^{2}$, and is therefore negligible. We can therefore immediately conclude that the electron cannot gain significant energy in this configuration. This reinforces a conclusion reached in previous work ${ }^{10}$, where it was concluded that this configuration would require some additional elements in order for net energy gain to be achieved. Furthermore, we can 


\begin{tabular}{|c|c|c|c|c|c|}
\hline Run & $\begin{array}{c}n_{e} \\
\left(10^{27} \mathrm{~m}^{-3}\right)\end{array}$ & $\begin{array}{c}\tau_{L} \\
(\mathrm{fs})\end{array}$ & $\begin{array}{c}B_{e x t} \\
(\mathrm{~T})\end{array}$ & $\begin{array}{c}\text { Abs. at 400fs } \\
(\%)\end{array}$ & $\begin{array}{c}\max .\left(\left|p_{x}\right|\right) \\
\left(m_{e} c\right)\end{array}$ \\
\hline \hline $\mathrm{A}$ & 0.3 & 40 & 0 & 16.7 & 49.9 \\
\hline $\mathrm{B}$ & 0.3 & 40 & 1000 & 32.9 & 35.4 \\
\hline $\mathrm{C}$ & 0.2 & 10 & 0 & 50.4 & 35.6 \\
\hline $\mathrm{D}$ & 0.2 & 10 & 1000 & 71.9 & 43.5 \\
\hline
\end{tabular}

TABLE I. Values of parameters used in PIC simulations and observed energy absorption at $400 \mathrm{fs}$. Note that $a_{0}=5$ in these simulations.

report that we have used numerical integration to check a few particular cases, all of which verified this conclusion.

\section{PIC SIMULATIONS}

The analysis presented in the preceding sections has number of limitations. Obviously the orbit of the electron puts a limit on the radius of a laser spot required for the plane wave approximation to be valid. However there is also the issue of the collective effects, which in rea laser-plasma interactions will be non-negligible.

To address this second concern, we have carried out some 1D Particle-in-Cell simulations using the Centra Laser Facility's ELPS code. These simulations were run on a grid with 50000 cells that are each $5 \mathrm{~nm}$ wide. On this grid we set a hydrogenic plasma that was $40 \mu \mathrm{m}$ wide and whose electron/proton density ranged from 0.1 to $0.35 \times 10^{27} \mathrm{~m}^{-3}$, with an initial electron temperature of $4 \mathrm{keV}$ (protons are cold). The electrons are represented by 500 macroparticles per cell, and the protons by 125 macroparticles per cell. The calculations were run up to $400 \mathrm{fs}$.

The envelope function of the incident laser field was a Gaussian, $\propto \exp \left(-\left(x-x_{0}\right)^{2} /\left(2\left(c \tau_{L}\right)^{2}\right)\right)$, with $\tau_{L}$ being in the range 10-40 fs across the entire set of simulations. The magnitude of the fields was set so that the normalized vector potential, $a_{0}=5$, with the laser electric field linearly polarized in the $y$-direction. The wavelength of the incident laser pulse was set to $1 \mu \mathrm{m}$. In addition to the laser pulse we also initialized the simulation with $B_{y}=B_{\text {ext }}$ throughout the domain.

Here we will report on a few results from this parameter scan. In runs $\mathrm{A}$, and $\mathrm{B}$ the set-up was as described above but with $\tau_{L}$ set to $40 \mathrm{fs}$, and $n_{e}=0.3 \times 10^{27} \mathrm{~m}^{-3}$ However in A, $B_{\text {ext }}=0$, and in B, $B_{\text {ext }}=1000 \mathrm{~T}$. In runs $\mathrm{C}$ and $\mathrm{D}, \tau_{L}$ set to $10 \mathrm{fs}$, and $n_{e}=0.2 \times 10^{27} \mathrm{~m}^{-3}$. The values of $B_{\text {ext }}$ were $0 \mathrm{~T}$ for $\mathrm{C}$ and $1000 \mathrm{~T}$ for $\mathrm{D}$. The energy absorption (from laser fields) was calculated at $400 \mathrm{fs}$ in each case, and these results are shown in Table I. The energy absorption is defined as the fraction of energy removed from the incident laser field, and is calculated via the reduction in the field energy associated with $E_{y}$ and $B_{z}$.

What we see from Table I was a general trend across the entire parameter scan, namely that the energy ab- sorption was always higher when a strong magnetic field was present. The increases in absorption are really quite substantial. In the case of runs A and B, the addition of the 'external' magnetic field results in an increase in absorption of $97 \%$, i.e. essentially a factor of two improvement. In the case of $\mathrm{C}$ and $\mathrm{D}$, where the $0 \mathrm{~T}$ absorption is already quite high, there is still a $43 \%$ improvement in the energy absorption. This coincides with what we would expect from our analysis of the single particle problem in previous sections, namely that we would expect the presence of these strong magnetic fields to lead to substantial increases in the absorption of laser energy. This indicates that the enhanced dephasing mechanism has a profound effect on the absorption despite the presence of strong collective effects in this interaction.

\section{CONCLUSIONS}

In this paper we showed that net energy gain can occur in single plane wave interactions provided another field is present which increases the dephasing rate during the interaction. This could be done with a decelerating electric field parallel to the wavevector of the incident pulse, but this is not a very useful configuration. Configurations where a uniform magnetostatic field which is perpendicular to the wavevector are much more useful, and we have easily demonstrated net energy gain from these configurations.

We also found that there is an optimal magnetic field that delivers the highest energy retention by the laserirradiated electron. The corresponding strength is inversely proportional to the laser pulse duration. In our examples, the optimal fields are $280 \mathrm{~T}$ and $430 \mathrm{~T}$ (see Fig.s 4 and 8), which is within reach to existing laser facilities $^{43,44}$

Finally, we have presented 1D Particle-in-Cell simulations of laser pulses interacting with near-critical plasma slabs with and without strong magnetic fields (in the same direction as laser polarization). In these simulations we see very substantial increases in the absorption of laser energy, which strongly indicates that the enhanced dephasing mechanism is able to enhance net energy gain even in complex interactions where there are strong collective effects at play.

\section{ACKNOWLEDGEMENTS}

The research of A. Arefiev was supported by the DOE Office of Science under Grant No. DE-SC0018312.

${ }^{1}$ A. Pukhov, Z.-M. Sheng, and J. Meyer-ter Vehn, Physics of Plasmas 6, 2847 (1999)

${ }^{2}$ A. Pukhov, Rep.Prog.Phys. 66, 47 (2003)

${ }^{3}$ G.D.Tsakiris, C.Gahn, and V.K.Tripathi, Phys.Plasmas 7, 3017 $(2000)$

${ }^{4}$ A. V. Arefiev, B. N. Breizman, M. Schollmeier, and V. N. Khudik, Phys. Rev. Lett. 108, 145004 (2012). 
${ }^{5}$ A. V. Arefiev, V. N. Khudik, and M. Schollmeier, Physics of Plasmas 21, 033104 (2014).

${ }^{6}$ D. Stark, T. Toncian, and A. Arefiev, Physical Review Letters 116, 185003 (2016).

${ }^{7}$ A. G. Krygier, D. W. Schumacher, and R. R. Freeman, Physics of Plasmas (1994-present) 21, 023112 (2014)

${ }^{8}$ S. P. D. Mangles, B. R. Walton, M. Tzoufras, Z. Najmudin, R. J. Clarke, A. E. Dangor, R. G. Evans, S. Fritzler, A. Gopal, C. Hernandez-Gomez, W. B. Mori, W. Rozmus, M. Tatarakis, A. G. R. Thomas, F. S. Tsung, M. S. Wei, and K. Krushelnick, Phys. Rev. Lett. 94, 245001 (2005).

${ }^{9}$ N. Naseri, D. Pesme, W. Rozmus, and K. Popov, Phys. Rev Lett. 108, 105001 (2012)

${ }^{10}$ A. P. L. Robinson, A. V. Arefiev, and D. Neely, Phys. Rev. Lett. 111, 065002 (2013).

${ }^{11}$ J. L. Shaw, F. S. Tsung, N. Vafaei-Najafabadi, K. A. Marsh, N. Lemos, W. B. Mori, and C. Joshi, Plasma Physics and Controlled Fusion 56, 084006 (2014).

${ }^{12}$ X. Zhang, V. N. Khudik, and G. Shvets, Physical Review Letters 114, 184801 (2015).

${ }_{13}^{13}$ A. G. York, H. M. Milchberg, J. P. Palastro, and T. M. Antonsen, Physical Review Letters 100, 195001 (2008).

${ }^{14}$ A. P. L. Robinson, A. V. Arefiev, and V. N. Khudik, Physics of Plasmas 22, 083114 (2015)

${ }^{15}$ A. V. Arefiev, V. N. Khudik, A. P. L. Robinson, G. Shvets, and L. Willingale, Physics of Plasmas 23, 023111 (2016).

${ }^{16}$ T. W. Huang, A. P. L. Robinson, C. T. Zhou, B. Qiao, B. Liu, S. C. Ruan, X. T. He, and P. A. Norreys, Physical Review E 93 063203 (2016)

${ }^{17}$ D. Umstadter, Journal of Physics D: Applied Physics 36, R15 (2003).

${ }^{18}$ P. Sprangle, E. Esarey, and J. Krall, Physics of Plasmas 3, 2183 (1996).

${ }^{19}$ S. P. Hatchett, C. G. Brown, T. E. Cowan, E. A. Henry, J. S. Johnson, M. H. Key, J. A. Koch, A. B. Langdon, B. F. Lasinski, R. W. Lee, A. J. Mackinnon, D. M. Pennington, M. D. Perry, T. W. Phillips, M. Roth, T. C. Sangster, M. S. Singh, R. A. Snavely, M. A. Stoyer, S. C. Wilks, and K. Yasuike, Physics of Plasmas 7, 2076 (2000)

${ }^{20}$ B.Liu, H.Y.Wang, J.Liu, B.Fu, Y.J.Xu, X.Q.Yan, and X.T.He, Phys. Rev. Lett. 110, 045002 (2013)

${ }^{21}$ S. A. Gaillard, T. Kluge, K. A. Flippo, M. Bussmann, B. Gall, T. Lockard, M. Geissel, D. T. Offermann, M. Schollmeier, Y. Sentoku, and T. E. Cowan, Physics of Plasmas 18, 056710 (2011) ${ }^{22}$ H.Liu, X.T.He, and S.G.Chen, Phys. Rev. E 69, 066409 (2004) ${ }^{23}$ L. Willingale, A. G. R. Thomas, P. M. Nilson, H. Chen, J. Cobble, R. S. Craxton, A. Maksimchuk, P. A. Norreys, T. C. Sangster, R. H. H. Scott, C. Stoeckl, C. Zulick, and . K. Krushelnick, New Journal of Physics 15, 025023 (2013).

${ }^{24}$ T. Kluge, S. A. Gaillard, K. A. Flippo, T. Burris-Mog, W. Enghardt, B. Gall, M. Geissel, A. Helm, S. D. Kraft, T. Lockard, J. Metzkes, D. T. Offermann, M. Schollmeier, U. Schramm, K. Zeil, M. Bussmann, and T. E. Cowan, New Journal of Physic 14, 023038 (2012)

${ }^{25}$ K. D. Xiao, T. W. Huang, L. B. Ju, R. Li, S. L. Yang, Y. C. Yang, S. Z. Wu, H. Zhang, B. Qiao, S. C. Ruan, C. T. Zhou, and X. T. He, Physical Review E 93, 043207 (2016).

${ }^{26}$ T. W. Huang, C. T. Zhou, A. P. L. Robinson, B. Qiao, A. V. Arefiev, P. A. Norreys, X. T. He, and S. C. Ruan, Physics of
Plasmas 24, 043105 (2017).

${ }^{27}$ E. Esarey, P. Sprangle, and J. Krall, Physical Review E 52, 5443 (1995).

${ }^{28}$ T. Häuser, W. Scheid, and H. Hora, Physics Letters A 186, 189 (1994)

29 J.-H. Yang, R. S. Craxton, and M. G. Haines, Plasma Physics and Controlled Fusion 53, 125006 (2011).

${ }^{30}$ V. Marceau, C. Varin, T. Brabec, and M. Piché, Physical Review Letters 111, 224801 (2013).

${ }^{31}$ P.-L. Fortin, M. Piché, and C. Varin, Journal of Physics B: Atomic, Molecular and Optical Physics 43, 025401 (2009).

${ }^{32}$ L. J. Wong and F. X. Kärtner, Optics Express 18, 25035 (2010).

${ }^{33}$ C. Varin, S. Payeur, V. Marceau, S. Fourmaux, A. April, B. Schmidt, P.-L. Fortin, N. Thiré, T. Brabec, F. Légaré, J.-C. Kieffer, and M. Piché, Applied Sciences 3, 70 (2013).

${ }^{34}$ S. Carbajo, E. A. Nanni, L. J. Wong, G. Moriena, P. D. Keathley, G. Laurent, R. D. Miller, and F. X. Kärtner, Physical Review Accelerators and Beams 19, 021303 (2016).

${ }^{35} \mathrm{M}$. Eloy, A. Guerreiro, J. T. Mendonça, and R. Bingham, Journal of Plasma Physics 73, 635 (2007).

${ }^{36}$ T. Plettner, R. L. Byer, E. Colby, B. Cowan, C. M. S. Sears, J. E. Spencer, and R. H. Siemann, Physical Review Special Topics Accelerators and Beams 8, 121301 (2005).

${ }^{37}$ M. Thévenet, A. Leblanc, S. Kahaly, H. Vincenti, A. Vernier, F. Quéré, and J. Faure, Nature Physics 12, 355 (2016)

${ }^{38}$ L. M.-W., H. C.-Y., L. Y.-L., C. S.-H, and I. Jovanovic, Physics of Plasmas 23, 123110 (2016)

${ }^{39}$ A. P. L. Robinson and A. V. Arefiev, Physics of Plasmas 24, 023101 (2017).

${ }^{40}$ A. P. L. Robinson and A. V. Arefiev, Physics of Plasmas 25, 053107 (2018).

${ }^{41}$ A. L. Troha, J. R. Van Meter, E. C. Landahl, R. M. Alvis, Z. A. Unterberg, K. Li, N. C. Luhmann, A. K. Kerman, and F. V. Hartemann, Physical Review E 60, 926 (1999).

${ }^{42}$ A. V. Arefiev, A. P. L. Robinson, and V. N. Khudik, Journal of Plasma Physics 81, 475810404 (2015)

${ }^{43}$ K. F. F. Law, M. Bailly-Grandvaux, A. Morace, S. Sakata, K. Matsuo, S. Kojima, S. Lee, X. Vaisseau, Y. Arikawa, A. Yogo, K. Kondo, Z. Zhang, C. Bellei, J. J. Santos, S. Fujioka, and H. Azechi, Applied Physics Letters 108, 091104 (2016), https://doi.org/10.1063/1.4943078.

${ }^{44}$ J. J. Santos, M. Bailly-Grandvaux, M. Ehret, A. V. Arefiev, D. Batani, F. N. Beg, A. Calisti, S. Ferri, R. Florido, P. ForestierColleoni, S. Fujioka, M. A. Gigosos, L. Giuffrida, L. Gremillet, J. J. Honrubia, S. Kojima, P. Korneev, K. F. F. Law, J.R. MarquÃšs, A. Morace, C. Moss ̃̃ (C), O. Peyrusse, S. Rose, M. Roth, S. Sakata, G. Schaumann, F. Suzuki-Vidal, V. T. Tikhonchuk, T. Toncian, N. Woolsey, and Z. Zhang, Physics of Plasmas 25, 056705 (2018), https://doi.org/10.1063/1.5018735. ${ }^{45}$ S.Fujioka and others, Sci.Rep. 3, 1170 (2013).

${ }^{46} \mathrm{~A}$. Arefiev, T. Toncian, and G. Fiksel, New Journal of Physics 18, 105011 (2016).

${ }^{47}$ C.Gahn et al., Phys.Rev.Lett. 83, 4772 (1999).

${ }^{48}$ Roberts and Buchsbaum, Phys.Rev. 135, A381 (1964).

${ }^{49}$ J.Angus and S.Krashennikov, Phys.Plasmas 16, 113103 (2009).

${ }^{50}$ A. V. Arefiev, G. E. Cochran, D. W. Schumacher, A. P. L. Robinson, and G. Chen, Physics of Plasmas 22, 013103 (2015). 


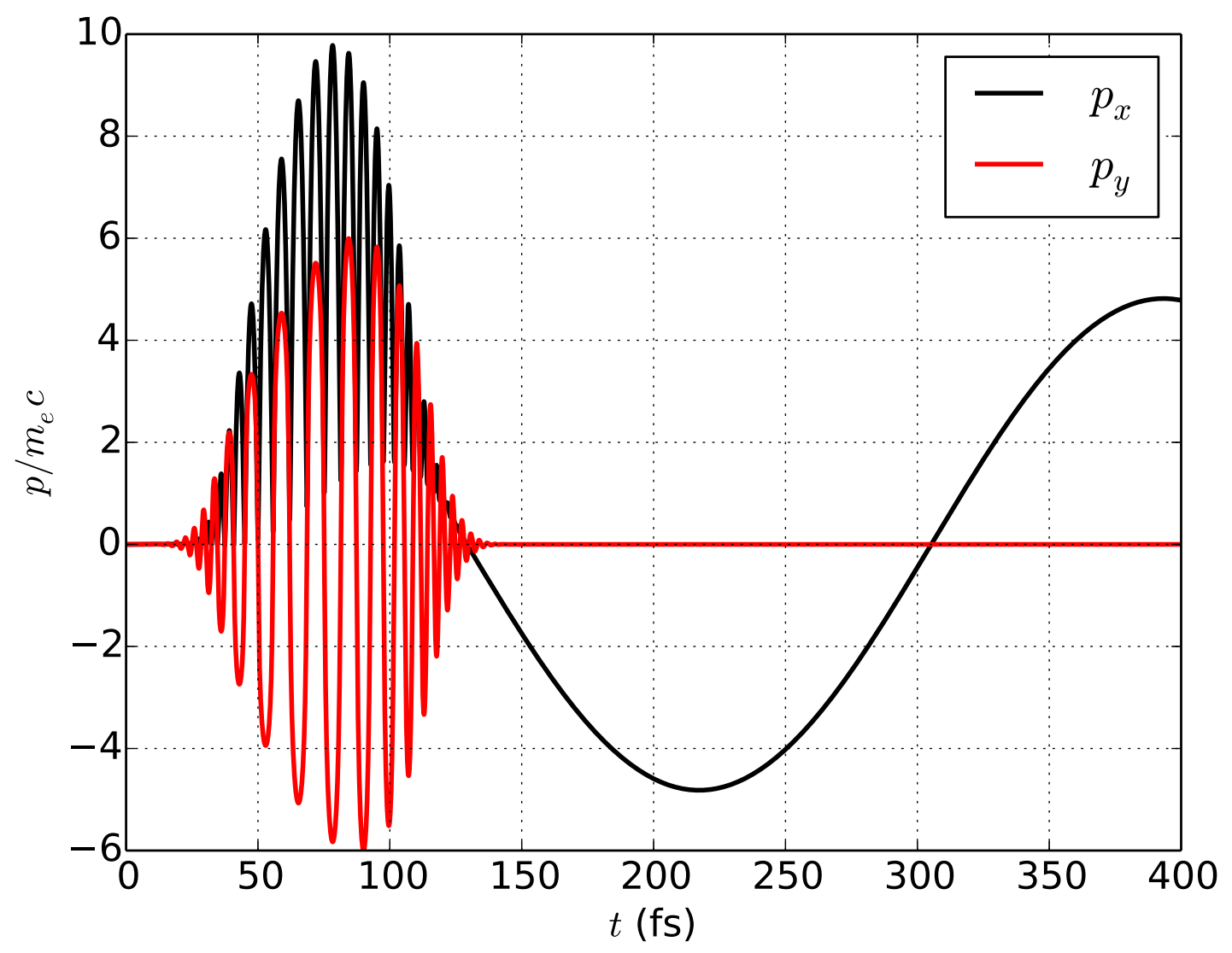

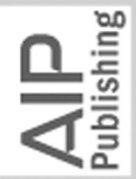




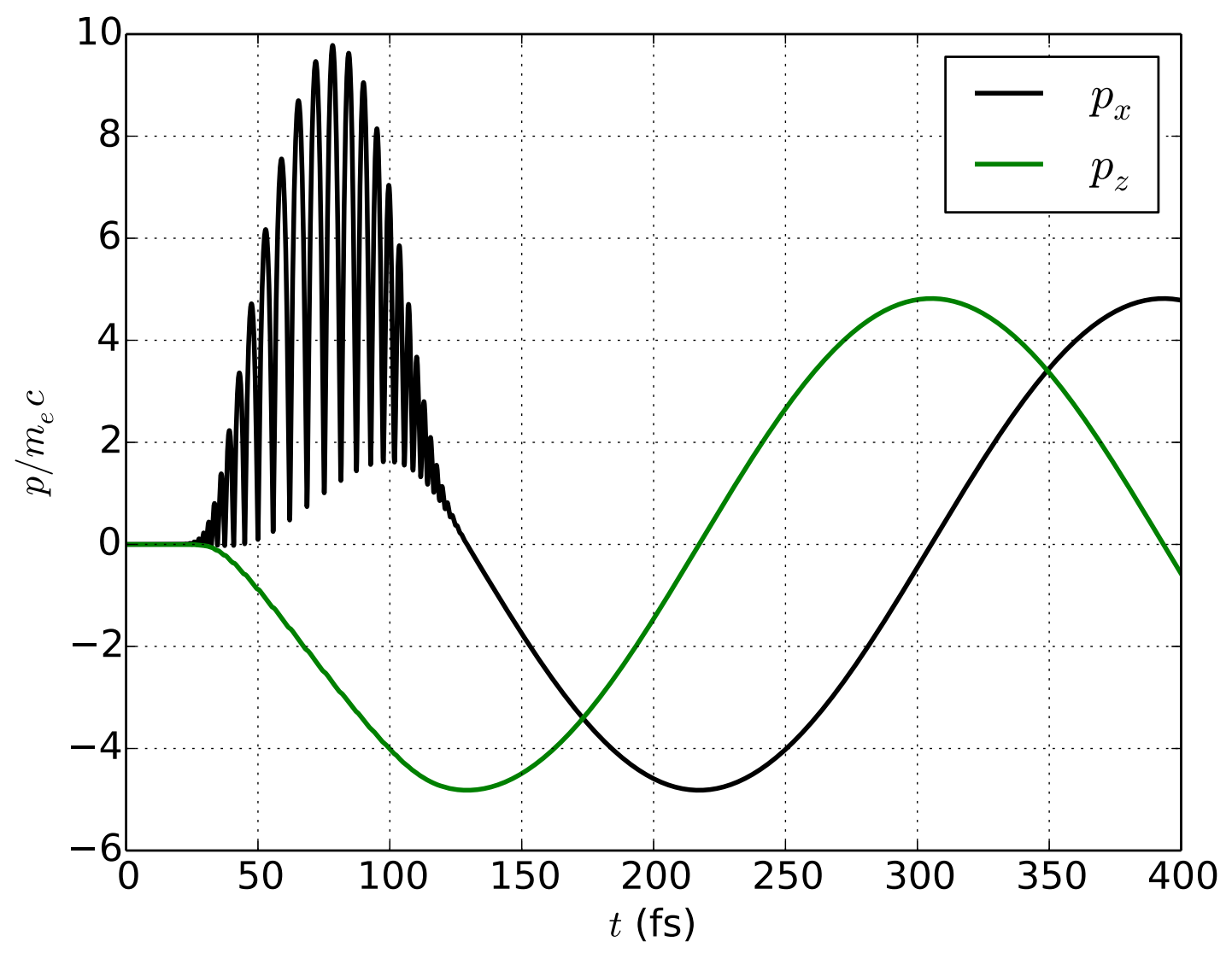

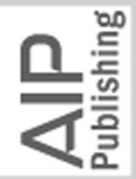




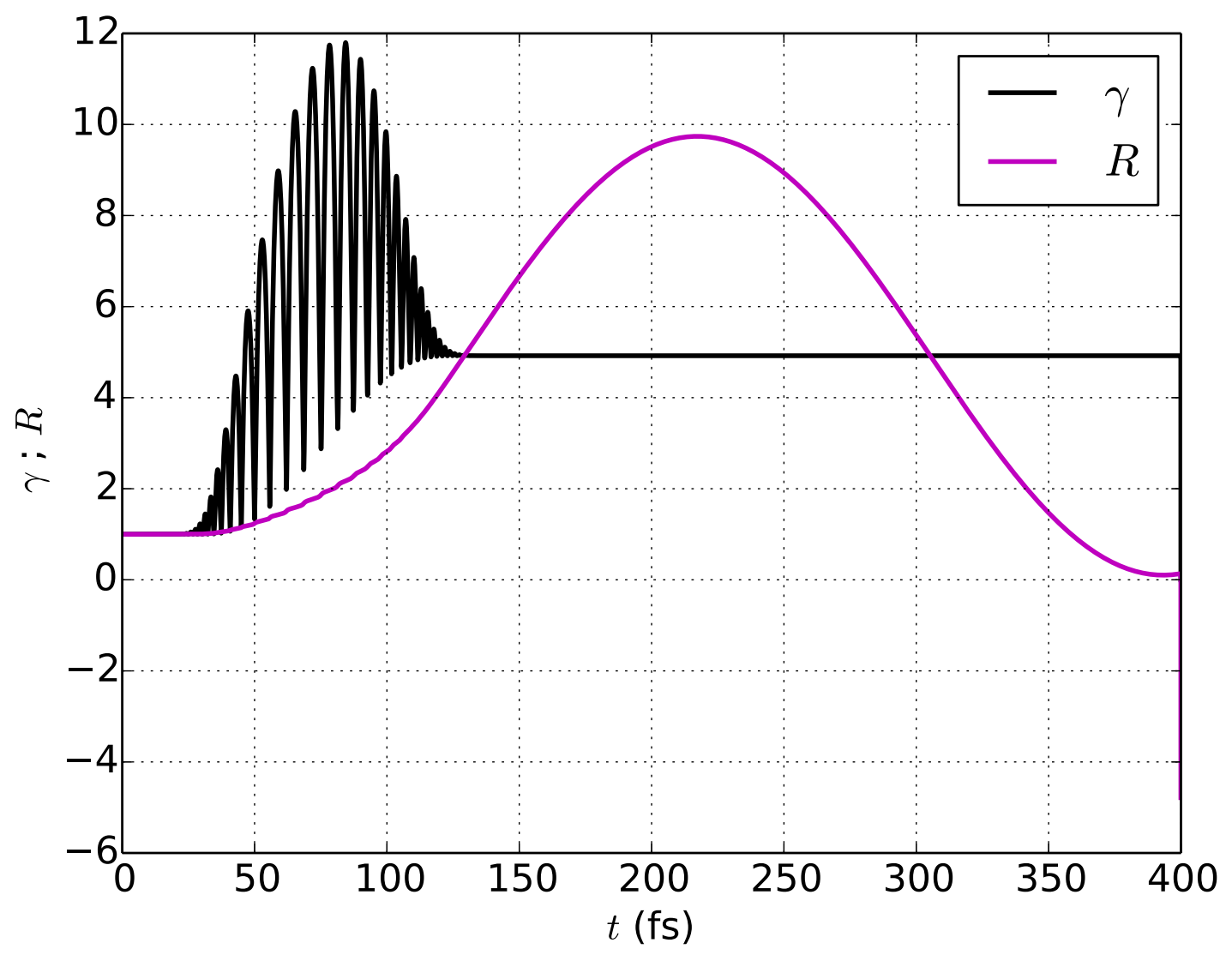

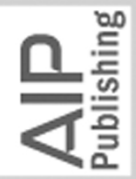




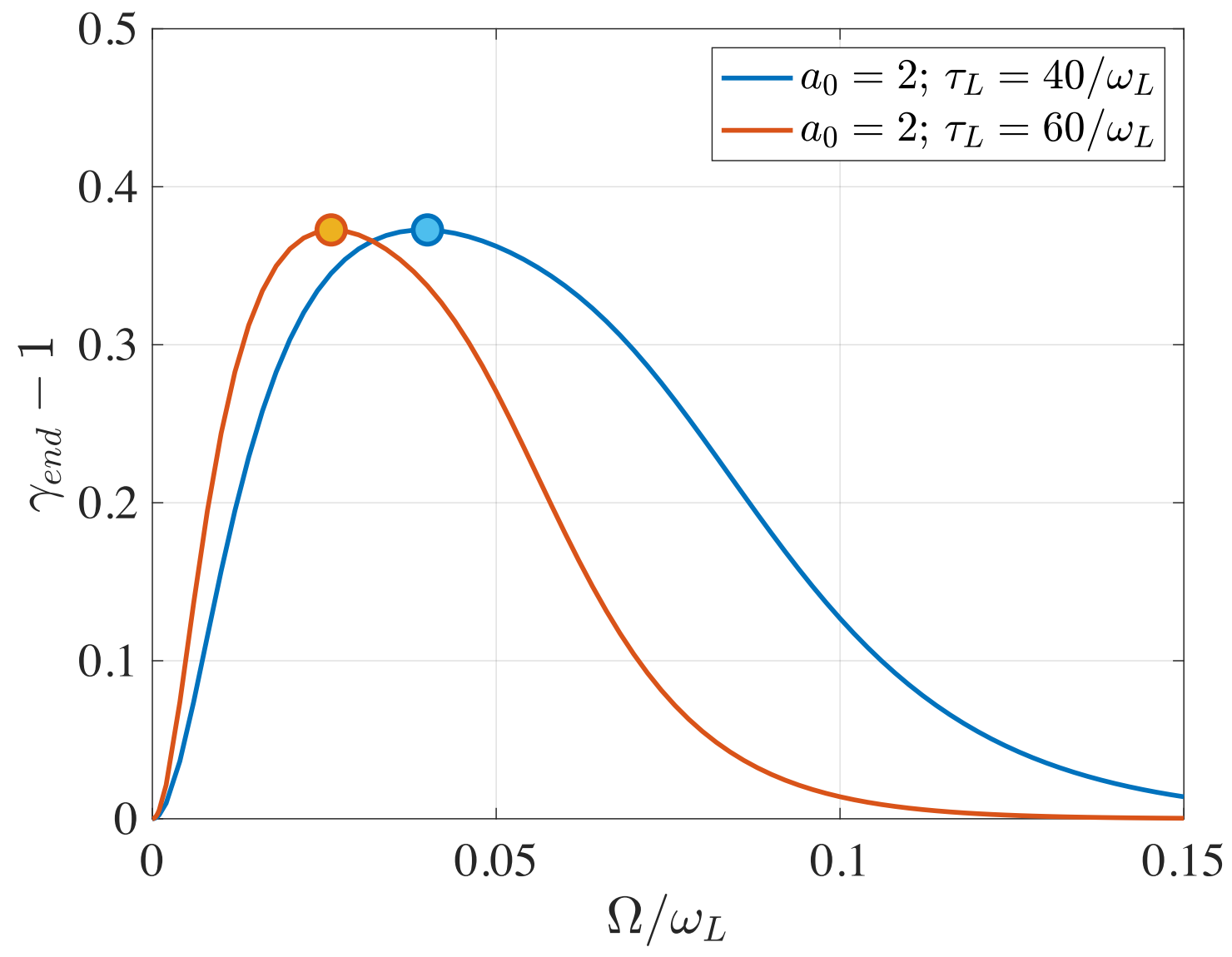



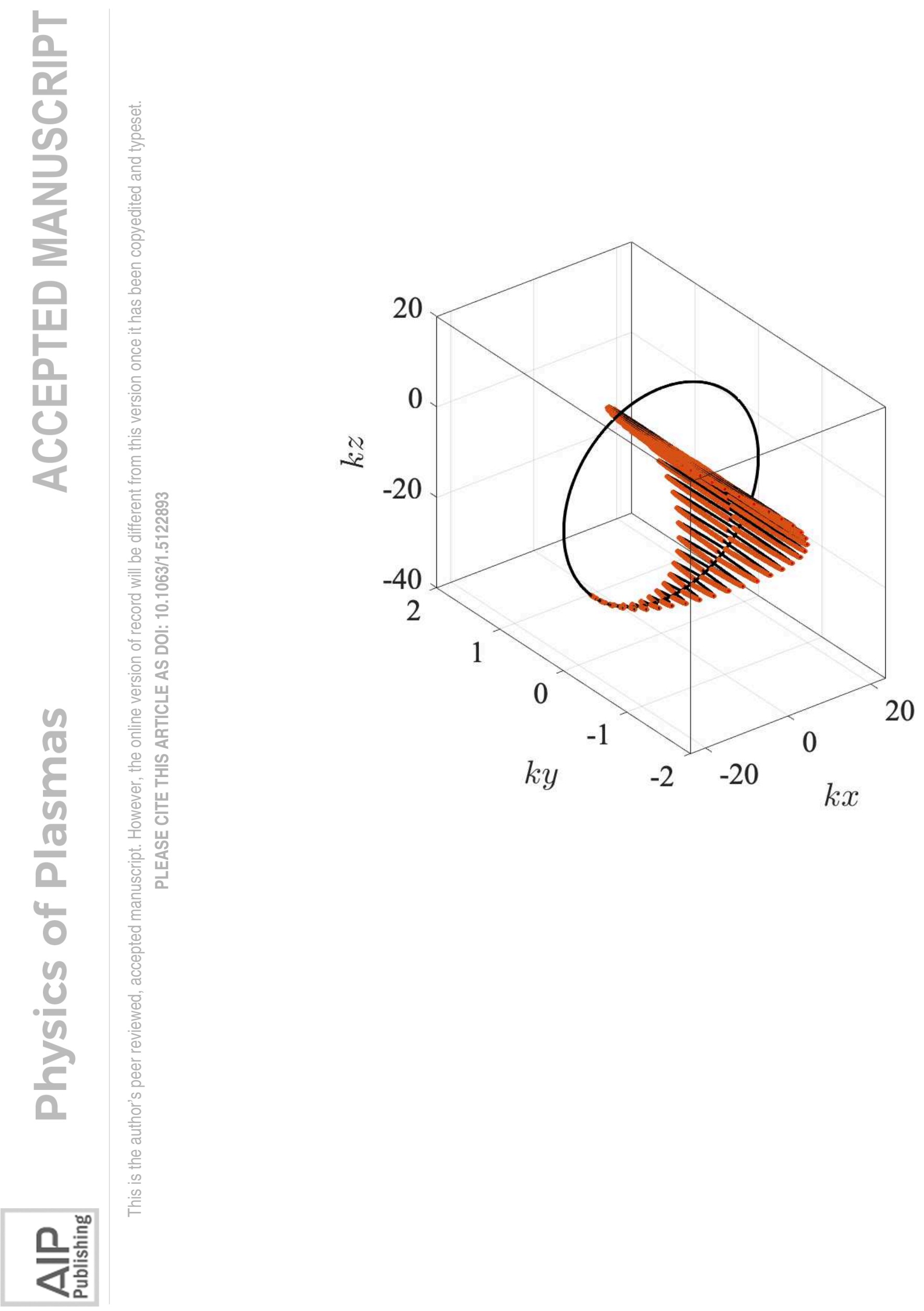

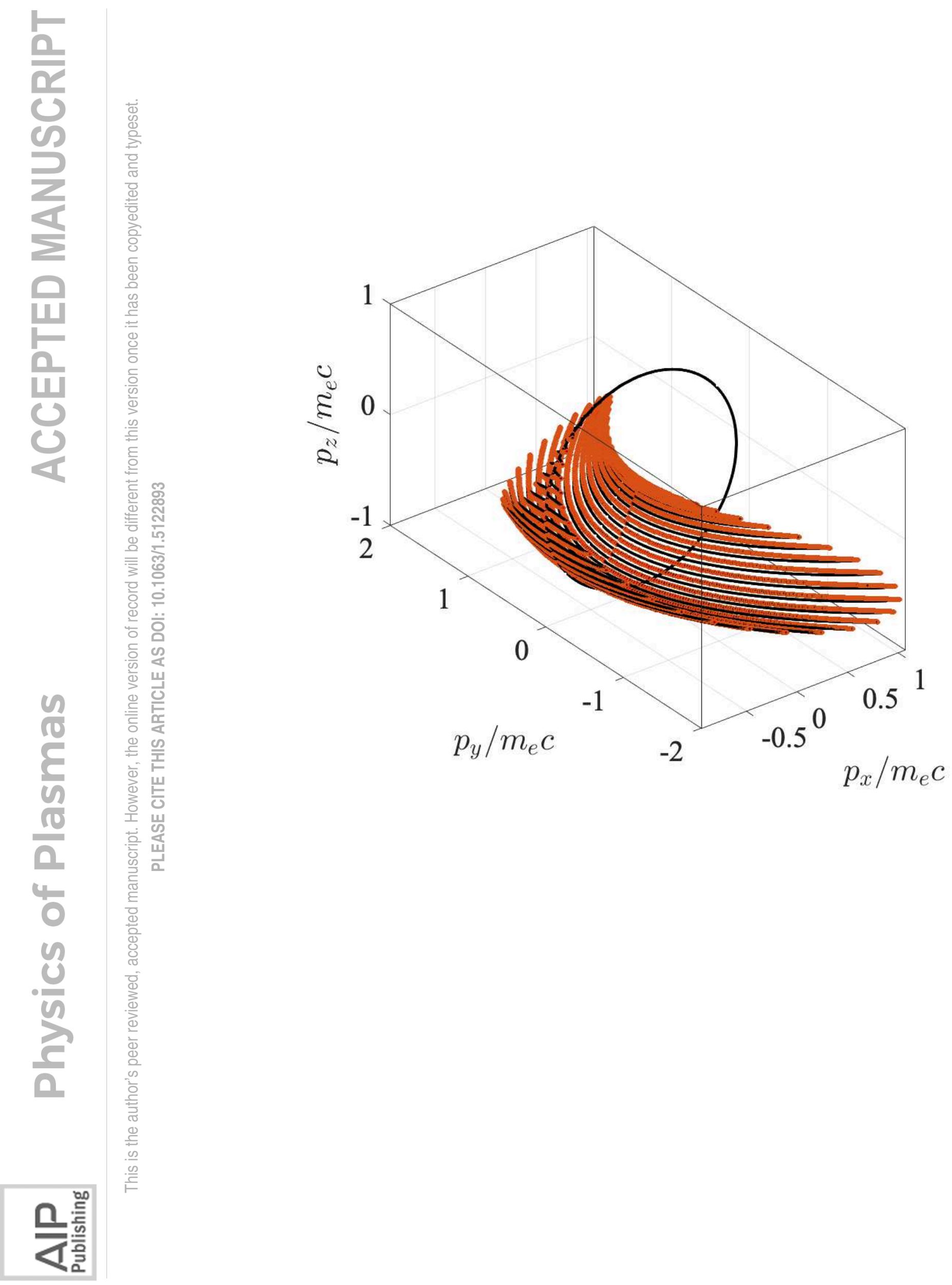


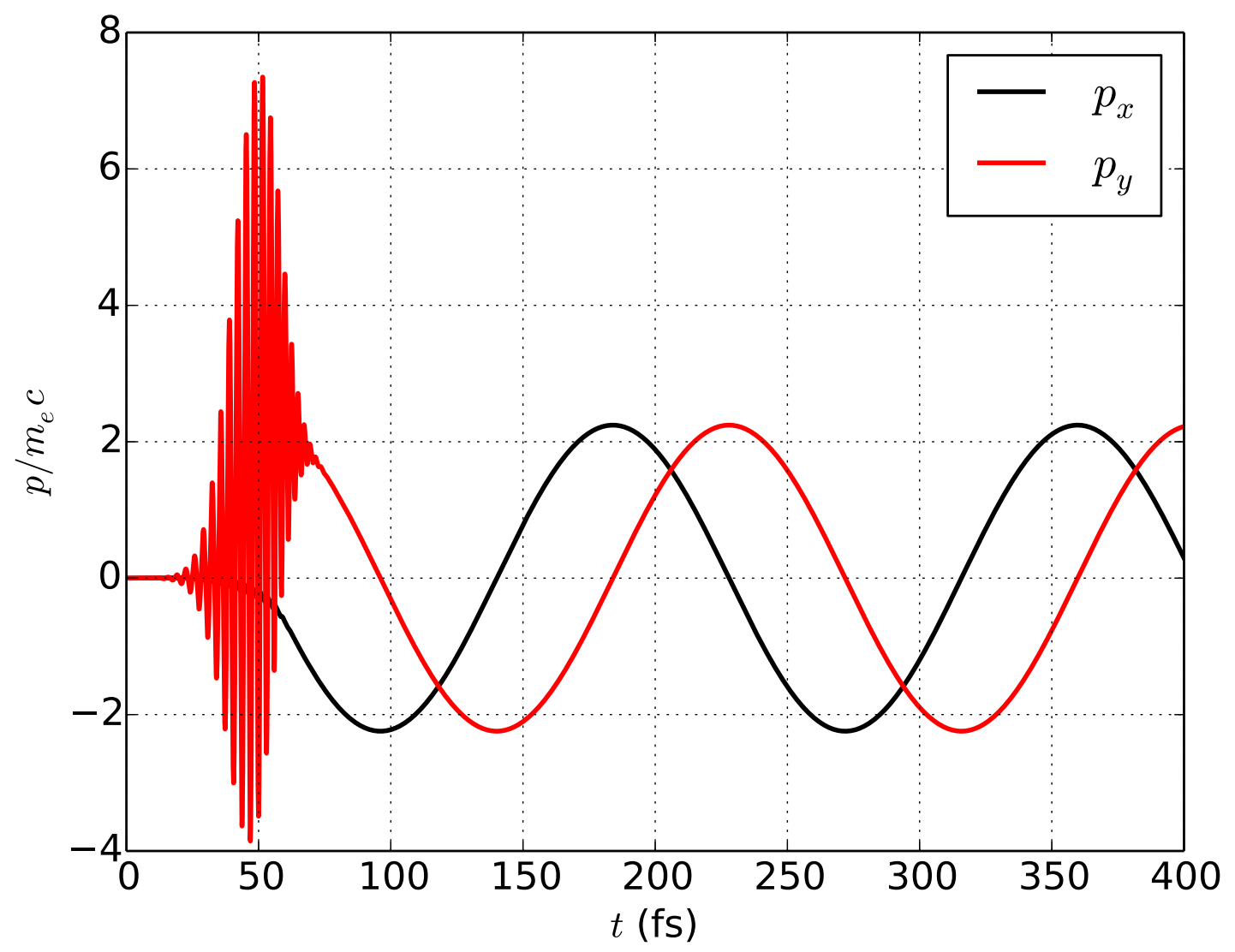

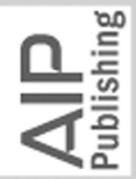




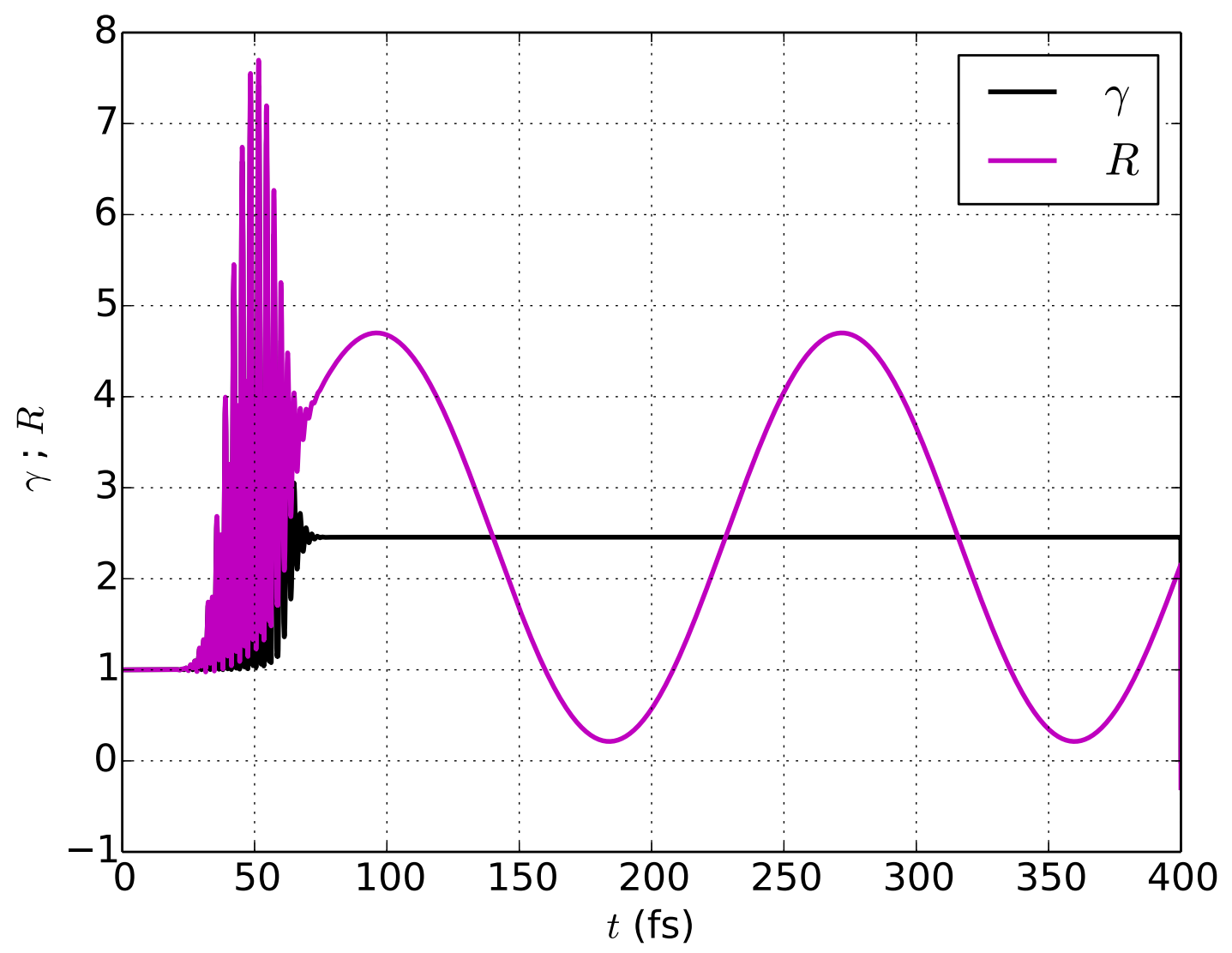

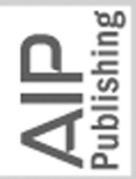




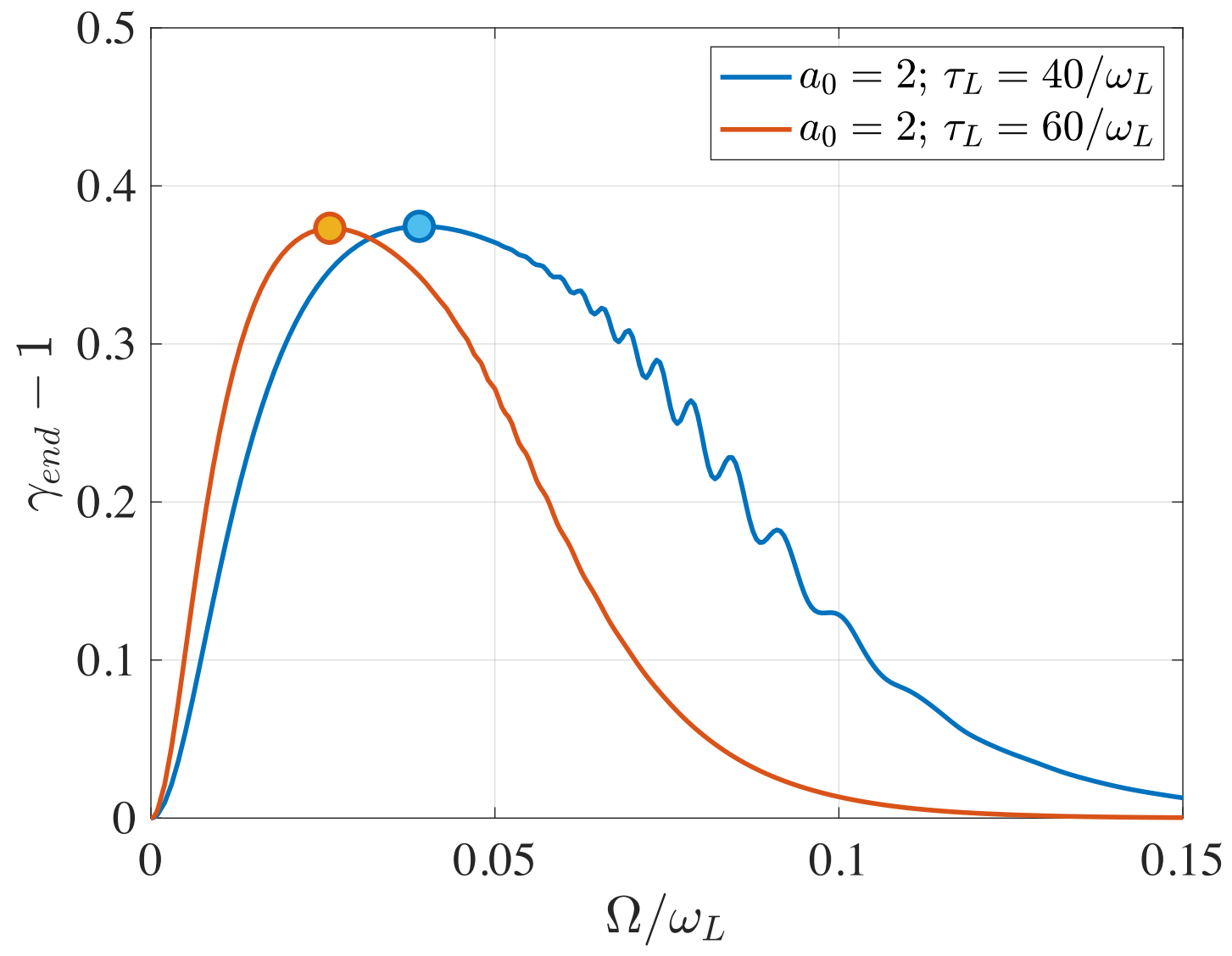




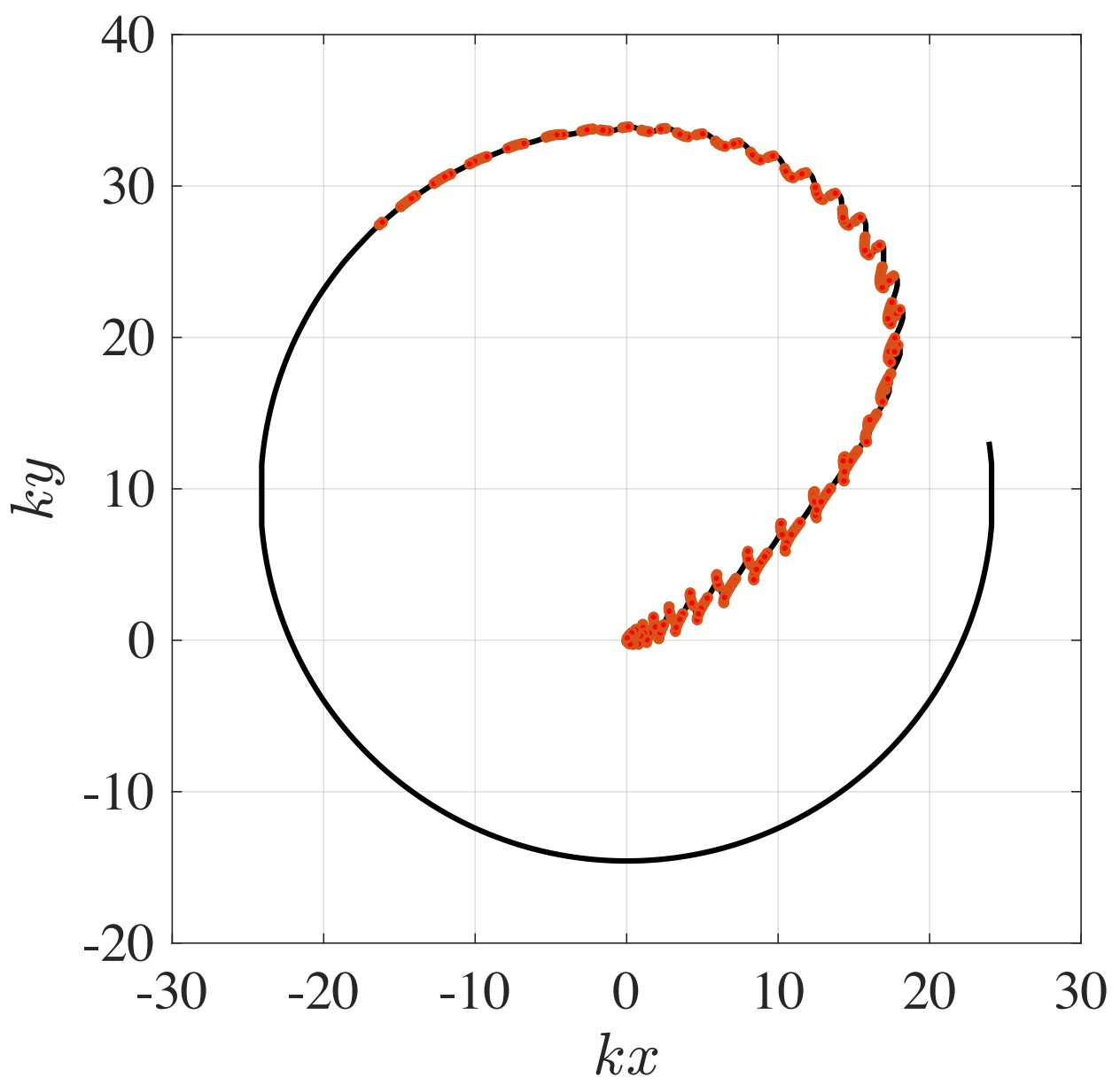

兄耪言 


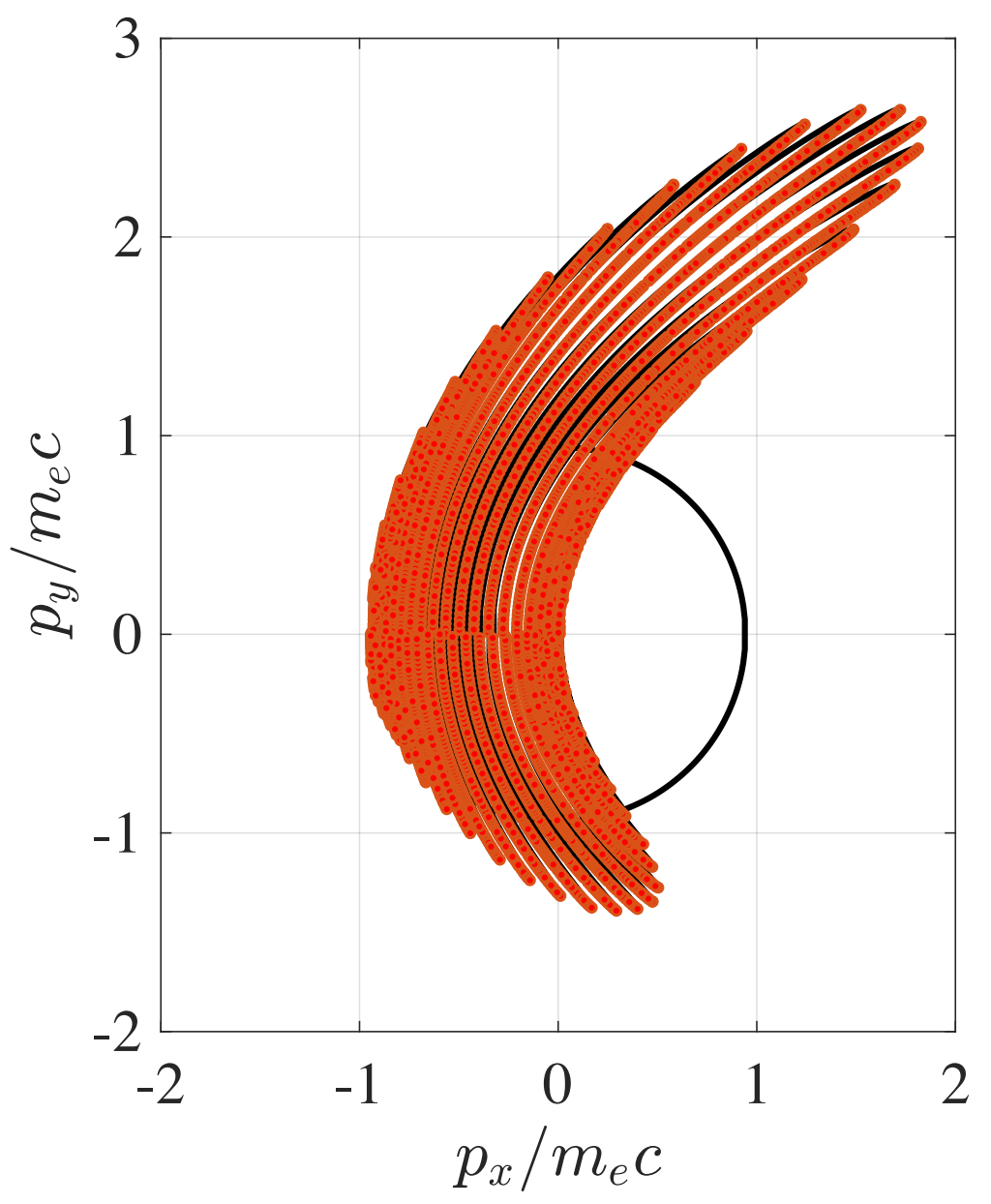

